\title{
Successful outcome in a patient with idiopathic dilated cardiomyopathy with bad obstetric history with no live issue
}

\author{
Renuka Malik*, Preeti Sharma
}

Department of Obstetrics \& Gynaecology, PGIMER, Dr. RML Hospital, New Delhi, India

Received: 29 February 2016

Accepted: 07 March 2016

\section{*Correspondence:}

Dr. Renuka Malik,

E-mail: renucam@yahoo.co.in

Copyright: $\odot$ the author(s), publisher and licensee Medip Academy. This is an open-access article distributed under the terms of the Creative Commons Attribution Non-Commercial License, which permits unrestricted non-commercial use, distribution, and reproduction in any medium, provided the original work is properly cited.

\begin{abstract}
Primary dilated cardiomyopathy is rare in women of childbearing age. Pregnancy in dilated cardiomyopathy carries high risk and can have adverse feto-maternal outcome even death. Traditionally patients of dilated cardiomyopathy with poor cardiac reserve are advised against pregnancy or termination of pregnancy in first trimester. A 26 year old patient with 5 abortions and no live issue diagnosed with Idiopathic dilated cardiomyopathy at 30 weeks at echocardiography (ECHO) with left ventricular ejection fraction (LVEF) of $25 \%$ (WHO class IV). She had no cardiac consultation earlier than in this pregnancy, though her earlier abortions were in hospital. Patient was kept with close monitoring. Dopplers parameters showed continued worsening. An emergency caesarean was done because of absent flow in ductus venosus at 34 weeks and a live child of $2.010 \mathrm{~kg}$ delivered. To date both mother and child are doing well. Awareness of such condition and a multidisciplinary approach is required in management of cardiomypathy in pregnancies, whose symptoms mimic that of normal pregnancy and can have adverse feto-maternal outcome.
\end{abstract}

Keywords: Idiopathic dilated cardiomyopathy, Peripartum cardiomyopathy

\section{INTRODUCTION}

Cardiovascular disease (CVD) complicates $1 \%$ of all pregnancies. ${ }^{1}$ It has surpassed hemorrhage, hypertensive disorders, and embolism to become the leading cause of indirect maternal death in pregnancy, accounting for approximately $26.4 \%$ of pregnancy-related deaths, in developed nations. ${ }^{1}$ In the future, the number of pregnant women with CVD is likely to rise as advances in health continue. Cardiomyopathy can rarely be encountered in pregnancy. Pregnancy in dilated cardiomyopathy carries high risk and can have adverse feto-maternal outcome. Traditionally patients of dilated cardiomyopathy, with poor cardiac reserve are advised against pregnancy or termination in first trimester. High degree of awareness of such conditions is necessary and multi-disciplinary approach is required in management of such conditions, which can easily be overlooked as symptoms overlap that of normal pregnancy.

\section{CASE REPORT}

A 26 year old, gravida 6 with 5 abortions, no living child was referred to us from a Government hospital at 30 weeks pregnancy, on 24.11.15 with an echo report of dilated cardiomyopathy with LVEF (left ventricular ejection fraction) of $25 \%$. Patient gave history of fatigue, dyspnea and palpitations since early pregnancy. She had history of 4 first trimester losses and one second trimester loss with no live issue, all in hospital. She was investigated for recurrent abortion from the referring hospital. She was not anemic. Serum TSH was 6 in first trimester for which she was put on 50ug thyroxine Rest all investigations including Glucose challenge test, TORCH, APLA (antiphospholipid antibody) and HIV were negative on detailed questioning she gave history of fatigue and dyspnea in previous pregnancies also, for which she did not have any record or any investigations done. There was no significant family history of cardiac 
disease or any cardio toxic drug intake. She had been put on injection claxane and ecospirin because of recurrent abortions since 10 weeks and had been hospitalized since then. When she had persistent tachycardia during hospitalization in the referring hospital at 28 weeks, a medical consultation was taken and an ECHO was done which showed dilated cardiomyopathy LVEF of $25 \%$ and she was referred to our centre. When patient was first seen in our hospital she was in NYHA class 2, WHO class IV. Her pulse was 120 beats/min, BP was 110/50, RR-20. She had moderate pedal edema. There was no hepatomegaly. P/A-fundal height was corresponding to 30 weeks, AGA (appropriate for gestational age), with live cephalic fetus. She was sent for cardiology consultation and was hospitalized in the obstetrical ward.
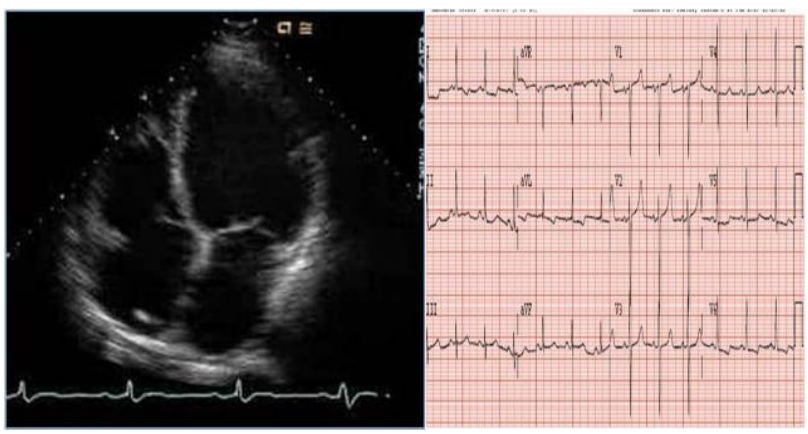

Figure 1: ECHO cardiograph showing dilated atria and ventricles with hypokinesia and LVEF of $25 \%$ and 12 lead ECG at 30 weeks of pregnancy.

Repeat ECG and ECHO (Figure 1) done by cardiologist confirmed the diagnoses of idiopathic dilated cardiomyopathy with LVEF of $25 \%$ with mild MR. She was put on lasix $40 \mathrm{mg}$ bd, digoxin $0.25 \mathrm{mg}$ od, betablocker $40 \mathrm{mg}$ bd and inj enoxaparin continued in dose of $40 \mathrm{ug} \mathrm{s} / \mathrm{c}$. She was kept propped up and her $\mathrm{sPO}_{2}$ (oxygen saturation) remained $98-100 \%$ on intermittent $\mathrm{O}_{2}$. A betamethasone cover was given in obstetrical ward and patient was kept under daily close monitoring by combined teams of cardiologist and obstetrician. She was kept on biweekly doppler flow assessment for fetal surveillance. Patient had persistent tachycardia, beta blockers was increased to three times. One week later, at 33 weeks period of gestation, her Doppler flow indices started worsening with s/d ratio in umbilical artery of 55.5.Ecospirin was stopped and at $33+5$ days she showed absent flow in ductous venosus. Injection enoxaparin was omitted and caesarean section was done after 12 hours, on 14.12.15 under combined spinal and epidural anaesthesia, keeping defibrillator ready, with central venous line and radial artery cannula placed for cardiac pressure recording and a $2.010 \mathrm{~kg}$ female baby was delivered and shifted to nursery. LNG-IUCD was inserted following delivery of placenta, for future contraception. Patent was shifted to ICU and epidural catheter was kept for 12 hours for pain relief and removed before next dose of enoxaparin. Patient remained stable. Her cardiac medications continued. She was shifted out to obstetrical ward on $4^{\text {th }}$ day of LSCS and allowed to breastfeed. Injection enoxaparin was continued for 10 days and she was discharged on 14 postoperative days. Her repeat echo showed same findings of dilated cardiomyopathy with EF of $30 \% 6$ weeks after delivery. Patient continues to be in follow up and is stable on medications and family support. She remains in NYHA class 2. She is a candidate for heart transplant in future.

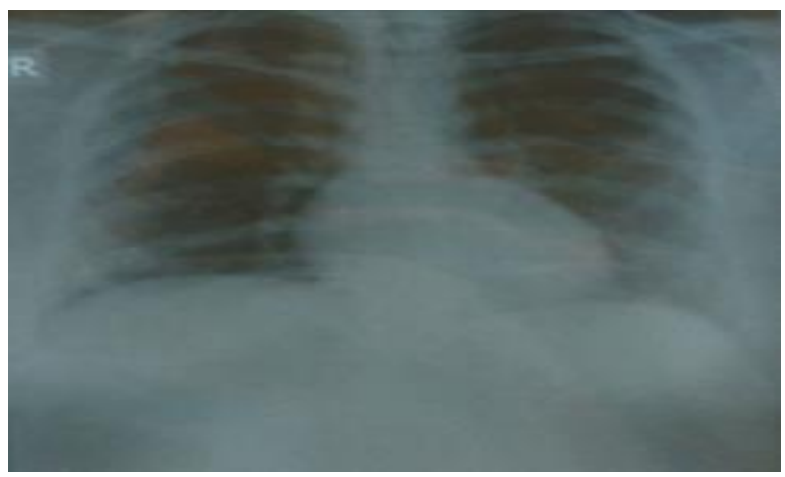

Figure 2: X-ray at 8 weeks following delivery showing persistent dilated heart.

\section{DISCUSSION}

Primary dilated cardiomyopathy is rare in women of childbearing age. It is more common in males. This patient was diagnosed for the first time in her sixth pregnancy on basis of persistent tachycardia and ECHO though her previous obstetrical mishaps were also in hospital. Hemodynamic changes occurring in pregnancy challenge the functional adaptability of cardiovascular system and unmask pre-existing cardiomyopathy. Cardiomyopathies may be classified as either primary or secondary, on the basis of etiology, or on the pathophysiology of the lesion: hypertrophic, dilated, or restrictive. $50 \%$ of dilated cardiomyopathy may be idiopathic in etiology, in which no cause is found. Other causes are Ischemic (7\%) hypertensive (4\%) Genetic, HIV (4\%), infiltrative (amyloidosis, sarcoidosis), infective (adenovirus, Coxsackievirus, cytomegalovirus, toxoplasmosis, Lyme disease), thyrotoxicosis, drug induced (1\%) and stress induced (tako-tsubo). Distinction is to be made with PPCM (peripartum cardiomyopathy) which is a distinct entity meeting all three criteria 1) - no pre-existing or identified cause for cardiac failure 2)LVEF <45\% 3). Occurring in last trimester to five months after delivery. ${ }^{2}$ Some believe that the definition of timing is too strict and that patients who develop symptoms of heart failure during the second or early third trimester should also be diagnosed with PPCM patients may be diagnosed for the first time as in our case or pregnancy may occur in a pre-diagnosed patient. ${ }^{3}$ Patients with LVEF less than $25 \%$ are advised against pregnancy. Pregnancy with dilated cardiomyopathy carries a risk of heart failure, cardio pulmonary arrest, cardiogenic shock, thromboembolism, maternal death and associated fetal complications like IUGR, prematurity and fetal death. 
Common complaints of a normal pregnancy such as palpitations, fatigue, decreased exercise tolerance and orthopnoea can often be identical to those with occult or overt heart disease. The normal physical examination in pregnancy can often mimic cardiac disease. Increased plasma volume might result in a systolic flow murmur (usually grade II/VI). Which can be heard in most of the normal pregnant patients. Moreover, distended neck veins, mild lower-extremity edema and tachycardia are normal findings in pregnancy. Estimation of BNP-B type natriuretic peptide can distinguish between normal pregnancy dyspnea and tachycardia with cardiac disease. It is a quick, within 20 min blood test which can diagnose $\mathrm{CHF}$, though ECHO is the confirmatory test. BNP at a cut point of $80 \mathrm{pg} / \mathrm{mL}$ was found to be highly sensitive and highly specific for the diagnosis of CHF. The negative predictive value of BNP values under $80 \mathrm{pg} / \mathrm{mL}$ was $98 \%$ for the diagnosis of $\mathrm{CHF}^{4}$ Hemodynamic changes that occur during pregnancy challenge the functional adaptability of the cardiovascular system in patients with pre-existing cardiomyopathies. During pregnancy, initially marked increases in circulating blood volume are met with an increase in stroke volume and a $15 \%$ to $20 \%$ increase in heart rate. The net effect is a $30 \%$ to $50 \%$ increase in cardiac output by the end of the first trimester, an effect that peaks between the second and third trimesters. During the early stages of pregnancy, increases in stroke volume are largely responsible for the observed increase in cardiac output, whereas later in pregnancy, an increased heart rate accounts for these changes. In addition there decreased systemic vascular resistance in second trimester created by the placenta, a phenomenon that continues until the $32^{\text {nd }}$ week of pregnancy when after load begins to rise again. During the third trimester, preload reduction might occur due to compression of the inferior vena cava by the gravid uterus, thus reducing cardiac output. Timing of delivery and mode of delivery is a critical decision in a cardiomyopathy patient which has to be individualized. Vaginal delivery has less risk of anesthetic complication and thromboembolism but should not be prolonged. On the other hand, operative delivery is planned and has the advantage of anesthetists.

Heart failure can occur during labour and following delivery cardiac output increases approximately 50\% in the second stage of labour. During delivery there is a catecholamine-induced increase in heart rate and stroke volume due to pain and anxiety in labour which have to be minimized by good anesthesia and cutting short second stage of labour and administration of diuretic following delivery of baby. During the peripartum period, there can be an increase of cardiac output of up to $30 \%$. Within the first hour of delivery, cardiac output might continue to increase to as much as $80 \%$ above pre-labour values due to the relief of IVC compression and potentially rapid auto transfusion from the placenta. Further alterations in the hemodynamic status occur most commonly within the first 12 to 24 hrs. post-partum. Therefore, women with pre-existing cardiomyopathies are at high risk for cardiac failure during delivery. ${ }^{5-7}$

\section{CONCLUSIONS}

Diagnosis of cardiomyopathy needs a high degree of awareness as the symptoms are same as which can pass as normal in pregnancy. ECHO is the gold standard for diagnosing heart disease. However, when ECHO cannot be done BNP levels which are quickly available can be done. Management of cardiomyopathy with pregnancy is equally critical. Multidisciplinary team round the clock is required including obstetrician, cardiologist and skilled anesthetist in such high risk patients and timing and mode of delivery have to be individualized.

\section{Funding: No funding sources Conflict of interest: None declared Ethical approval: Not required}

\section{REFERENCES}

1. Hameed AB, Stellar J. Cardiovascular disease in pregnancy: What ob/gyns need to know? Contemporary OB/GYN. 2015.

2. Givertz MM. Peripartum cardiomyopathy, circulation. 2013;127:e622-6.

3. Elkayam U, Akhter MW, Singh H, Khan S, Bitar F. Pregnancy-associated cardiomyopathy: clinical characteristics and a comparison between early and late presentation. Circulation. 2005;111:2050-5.

4. Maisel A. B-type natriuretic peptide levels: diagnostic and prognostic in congestive heart failure what's next? Circulation. 2002;105:2328-31.

5. Stergiopoulos K, Shiang E, Bench T. Pregnancy in patients with pre-existing cardiomyopathies. J Am Coll Cardiol. 2011;58:337-50.

6. Barbosa J, Feijão M, Carvalho F, Alencar C, Feitosa F. Idiopathic dilated cardiomyopathy in pregnancy. Open J Obstetr Gynecol. 2013;3:438-40.

7. Grewal J, Siu SC, Ross HJ. Pregnancy outcome in women with dilated cardiomyopathy. JACC. 2009;55(1):45-52.

Cite this article as: Malik $\mathrm{R}$, Sharma $\mathrm{P}$. Successful outcome in a patient with idiopathic dilated cardiomyopathy with bad obstetric history with no live issue. Int $\mathbf{J}$ Reprod Contracept Obstet Gynecol 2016;5:1225-7. 